\title{
Gauge boson couplings at LEP
}

\author{
S. Villa ${ }^{a}$ \\ ${ }^{a}$ Swiss Federal Institute of Technology, EPFL, LPHE-IPEP, 1015 Lausanne, Switzerland
}

A review is given of the measurements of triple and quartic couplings among the electroweak gauge bosons performed at LEP by the four experiments ALEPH, DELPHI, L3 and OPAL. Emphasis is placed on recently published results and on combinations of results performed by the LEP electroweak gauge-couplings group. All measurements presented are consistent with the Standard Model expectations.

\section{INTRODUCTION}

Self couplings among electroweak gauge bosons are a consequence of the non-Abelian structure of the Standard Model (SM) of electroweak interactions [1]. The measurement of triple (TGC) and quartic couplings (QGC) has been one of the main activities of the LEP experiments (ALEPH, DELPHI, L3 and OPAL) after the $\mathrm{e}^{+} \mathrm{e}^{-}$centreof-mass energy $(\sqrt{s})$ was raised above the threshold for the production of two $\mathrm{W}$ bosons (LEP2 phase). Experiments have performed both precision measurements of couplings as predicted by the theory and searches for anomalous couplings which could be present as a consequence of new physics. The theoretical input is normally expressed in the form of an effective Lagrangian containing all operators of a given order which contribute to the set of vertices under study and which are consistent with basic physical assumptions such as Lorentz and electromagnetic gauge invariance. The extraction of couplings is then performed either by fitting distributions of a chosen set of sensitive variables or by means of optimal observables techniques [2], in which the sensitivity to the couplings is concentrated in a reduced number of properly defined variables.

All results reported in this paper are based on data collected at LEP during 1996-2000 at $161 \mathrm{GeV}<\sqrt{s}<209 \mathrm{GeV}$, corresponding to total integrated luminosities up to about $700 \mathrm{pb}^{-1}$ per experiment. For many channels and couplings, LEP combined results exist. The latest combinations are summarized in 3] where reference is given to the individual publications. More recent results are not yet combined and will therefore be referenced directly in the following. The individual references should be consulted for details about experimental techniques and composition of data sets.

This document is organized as follows: Section 2 deals with charged triple couplings, i.e. couplings between the $\mathrm{W}$ and neutral bosons (WWZ and $\mathrm{WW} \gamma$ vertices), Section 3 describes searches for purely neutral TGCs, not foreseen in the SM, and finally Section 4 reports limits on QGCs.

\section{CHARGED TRIPLE GAUGE COU- PLINGS}

The WWZ and WW $\gamma$ vertices can be probed at LEP2 in WW, single $\mathrm{W}$ and single $\gamma$ production. To lowest order, three Feynman graphs contribute to WW production: the s-channel $\mathrm{Z}$ and $\gamma$ production and the t-channel neutrino exchange. The two s-channel diagrams contain the WWZ and $\mathrm{WW} \gamma$ vertices. $\mathrm{WW} \gamma$ is also involved in the single $\mathrm{W}$ process $\left(\mathrm{e}^{+} \mathrm{e}^{-} \rightarrow \mathrm{We} \nu\right)$ via $\mathrm{W}-\gamma$ fusion and in single $\gamma$ production $\left(\mathrm{e}^{+} \mathrm{e}^{-} \rightarrow \nu_{\mathrm{e}} \bar{\nu}_{\mathrm{e}} \gamma\right)$, via $\mathrm{W}$-boson fusion. The most general parametrization of the WWZ and WW $\gamma$ vertices can be written as a function of seven complex TGCs for each vertex [4. Some assumptions are normally made in order to reduce the number of parameters to be measured. Imposing the conservation of $\mathrm{C}$ and $\mathrm{P}$ symmetry and electromagnetic gauge invariance reduces the number of free pa- 
rameters to five, usually chosen to be $g_{1}^{\mathrm{Z}}, \kappa_{\gamma}$, $\lambda_{\gamma}, \kappa_{\mathrm{Z}}$ and $\lambda_{\mathrm{Z}}$. At tree level within the SM, $g_{1}^{Z}=\kappa_{\gamma}=\kappa_{\mathrm{Z}}=1$ and $\lambda_{\gamma}=\lambda_{\mathrm{Z}}=1$. A further reduction is normally achieved by the requirement of custodial $\mathrm{SU}(2)$ gauge invariance, which translates to the constraints $\kappa_{\mathrm{Z}}=g_{1}^{\mathrm{Z}}-\tan ^{2} \theta_{W}\left(\kappa_{\gamma}-1\right)$ and $\lambda_{\mathrm{Z}}=\lambda_{\gamma}$ [56], where $\theta_{W}$ is the weak mixing angle. $g_{1}^{\mathrm{Z}}, \kappa_{\gamma}$ and $\lambda_{\gamma}$ are normally chosen as the minimal set of parameters to be measured and are the ones used in the LEP-wide combinations.

The WW channel gives the best sensitivity at LEP in the measurement of charged TGCs. Anomalous couplings would affect the total production cross section, the production angle and the polarization of the $\mathrm{W}$ bosons. A WW event, yielding two fermions and two anti-fermions in the final state, is completely described in terms of five angles: the $\mathrm{W}$ production angle and the polar and azimuthal angles of the fermion in the $\mathrm{W}^{-}$rest frame and of the anti-fermion in the $\mathrm{W}^{+}$ rest frame. The distributions of these angles, or other quantities derived from them, are used by the LEP experiments to derive measurements of TGCs. As an example, the distribution of the cosine of the $\mathrm{W}^{-}$production angle in fully hadronic WW events collected by L3 [7] is shown in Figure 1 where the effect of anomalous values of $g_{1}^{Z}$ is also plotted.

The single $\mathrm{W}$ channel gives a sensitivity comparable to that obtained with WW events only on $\kappa_{\gamma}$; on the other hand it gives the possibility to disentangle the effects of the WWZ and $\mathrm{WW} \gamma$ vertices, which are correlated in measurements based on the WW channel. In this process, the electron is produced at very low polar angle and is therefore not detected, leaving a signature of only one $\mathrm{W}$ boson in the detector. LEP experiments use different kinematic variables to extract the couplings from the single $\mathrm{W}$ channel. As an example the transverse momentum of the electrons and muons in leptonically decaying W's as measured by ALEPH are shown in Figure 2 The energy spectrum and the angular distribution of the photon produced in single $\gamma$ processes are sensitive to the $\mathrm{WW} \gamma$ vertex, even though less than the WW and single $\mathrm{W}$ channels.

Measurements of charged TGCs by the four LEP experiments are summarized in Table 1 to-

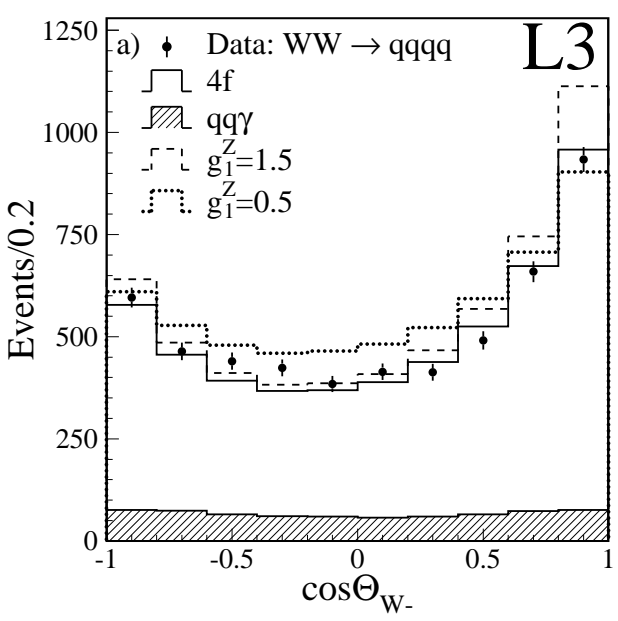

Figure 1. Distribution of the $\mathrm{W}^{-}$production angle in hadronic W-pair events collected by the L3 detector. Data are shown together with the expectations for the SM and for anomalous values of $g_{1}^{Z}$.

gether with their combination 3 . All results are obtained by varying one parameter while setting the others to their SM value. The constraints $\kappa_{\mathrm{Z}}=g_{1}^{\mathrm{Z}}-\tan ^{2} \theta_{W}\left(\kappa_{\gamma}-1\right)$ and $\lambda_{\mathrm{Z}}=\lambda_{\gamma}$ are imposed. The LEP combined results of fits to two of the three TGCs are shown in Figure B All measurements are consistent with the SM expectations.

\section{NEUTRAL TRIPLE GAUGE COU- PLINGS}

Triple couplings among neutral electroweak gauge bosons are not present in the SM at tree level, and get contributions of the order of $10^{-4}$ from loop diagrams. Such couplings could be enhanced by new physics effects [8] and it is therefore important to set limits on their strength. The $\gamma \gamma \mathrm{Z}, \gamma \mathrm{ZZ}$ and ZZZ vertices can give as on-shell final state bosons either $Z \gamma$ or ZZ. Two independent parametrizations describe the two cases: the eight $h_{i}^{\mathrm{V}}$ couplings $(i=1, \ldots, 4)$ for the $\mathrm{Z} \gamma$ final state and the four $f_{i}^{\mathrm{V}}$ couplings $(i=4,5)$ for the 
Table 1

Results of the measurements of charged TGCs by the LEP experiments and their combinations. Statistical and systematic uncertainties are included.

\begin{tabular}{lcrrrr}
\hline parameter & ALEPH & DELPHI & \multicolumn{1}{c}{ L3 } & \multicolumn{1}{c}{ OPAL } & \multicolumn{1}{c}{ LEP } \\
\hline$g_{1}^{Z}$ & $1.026_{-0.033}^{+0.034}$ & $1.002_{-0.040}^{+0.038}$ & $0.928_{-0.041}^{+0.042}$ & $0.985_{-0.034}^{+0.035}$ & $0.991_{-0.021}^{+0.022}$ \\
$\kappa_{\gamma}$ & $1.022_{-0.072}^{+0.073}$ & $0.955_{-0.086}^{+0.090}$ & $0.922_{-0.069}^{+0.071}$ & $0.929_{-0.081}^{+0.085}$ & $0.984_{-0.047}^{+0.042}$ \\
$\lambda_{\gamma}$ & $0.012_{-0.032}^{+0.033}$ & $0.014_{-0.042}^{+0.044}$ & $-0.058_{-0.044}^{+0.047}$ & $-0.063_{-0.036}^{+0.036}$ & $-0.016_{-0.023}^{+0.021}$ \\
\hline
\end{tabular}
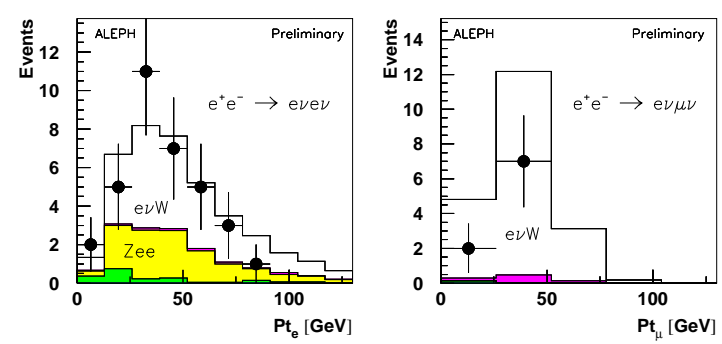

Figure 2. Distributions of the transverse momentum of electrons (left) and muons (right) in single $\mathrm{W}$ events collected by ALEPH.

ZZ final state 48, where in both sets $\mathrm{V}$ stands for the s-channel exchanged off-shell boson, which can be a $\mathrm{Z}$ or a $\gamma$.

The $\mathrm{Z} \gamma$ final state is produced at LEP by events in which the photon is radiated off the incoming electron or positron. The presence of anomalous couplings would increase the total cross section and enhance the photon production at large polar angles. By fitting distributions of kinematic variables such as the photon energy, polar angle and recoil mass, experiments are able to set limits on the $h_{i}^{\mathrm{V}}$ couplings. The LEP combined results [3] from fits where each parameter is measured separately while setting all others to zero, are summarized in Table 2 as 95\% confidence level (C.L.) limits. Final updated results have been published by L3 $[9$, which are not taken into account in this combination.

The ZZ final state is accessible at LEP2 via tchannel electron exchange. An s-channel $\gamma^{*} / \mathrm{Z}^{*}$ would yield the same final state if neutral $f_{4,5}^{\mathrm{V}}$
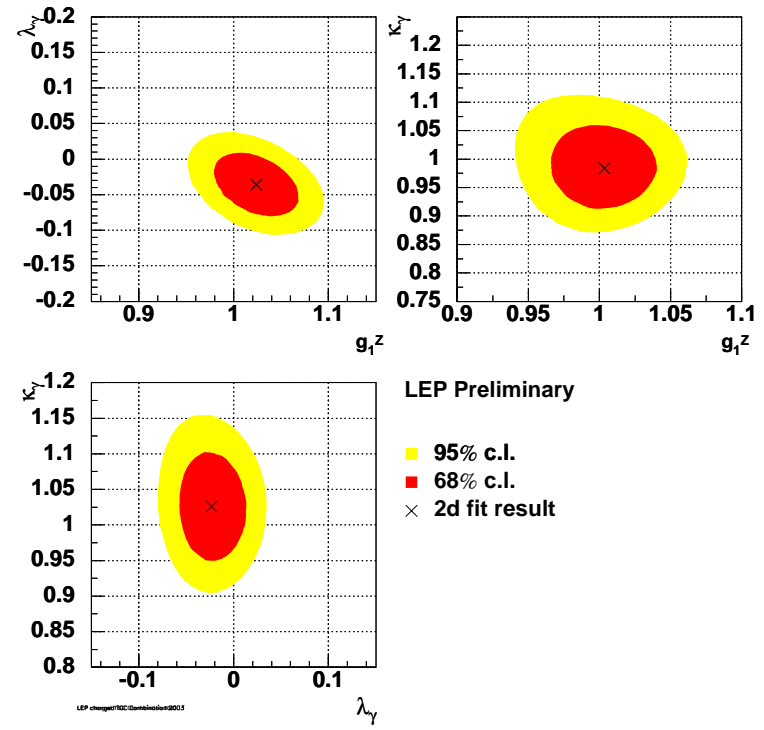

Figure 3. The $68 \%$ C.L. and $95 \%$ C.L. contours for two-parameter fits of charged TGCs (LEP combination). In each case, the third parameter is set to its SM value and the constraints $\kappa_{\mathrm{Z}}=g_{1}^{\mathrm{Z}}-\tan ^{2} \theta_{W}\left(\kappa_{\gamma}-1\right)$ and $\lambda_{\mathrm{Z}}=\lambda_{\gamma}$ are imposed.

TGCs were present. These anomalous couplings would increase the total cross section and modify the event kinematics. ZZ production has been studied at LEP in all of its visible topologies, and no deviations from the SM predictions have been observed. The measured ZZ production cross section as a function of $\sqrt{s}$ is plotted in Figure 4. where the effect of non-zero anomalous couplings is also shown. Combined LEP results on the $f_{4,5}^{\mathrm{V}}$ 
Table 2

Results of the combination of the measurements of neutral TGCs by the LEP experiments. Statistical and systematic uncertainties are included.

\begin{tabular}{cc}
\hline parameter & $95 \%$ C.L. \\
\hline$h_{1}^{\gamma}$ & {$[-0.056,+0.055]$} \\
$h_{2}^{\gamma}$ & {$[-0.045,+0.025]$} \\
$h_{3}^{\gamma}$ & {$[-0.049,-0.008]$} \\
$h_{4}^{\gamma}$ & {$[-0.002,+0.034]$} \\
$h_{1}^{\mathrm{Z}}$ & {$[-0.13,+0.13]$} \\
$h_{2}^{\mathrm{Z}}$ & {$[-0.078,+0.071]$} \\
$h_{3}^{\mathrm{Z}}$ & {$[-0.20,+0.07]$} \\
$h_{4}^{\mathrm{Z}}$ & {$[-0.05,+0.12]$} \\
$f_{4}^{\gamma}$ & {$[-0.17,+0.19]$} \\
$f_{4}^{\mathrm{Z}}$ & {$[-0.30,+0.30]$} \\
$f_{5}^{\gamma}$ & {$[-0.32,+0.36]$} \\
$f_{5}^{\mathrm{Z}}$ & {$[-0.34,+0.38]$} \\
\hline
\end{tabular}

couplings are summarized in Table 2 in the form of $95 \%$ C.L. intervals.

\section{QUARTIC GAUGE COUPLINGS}

Quartic couplings are present in the SM Lagrangian, but their size is expected to be too small to be measurable at LEP. The LEP experiments have therefore performed measurements to set limits on the existence of anomalous QGCs, both of the type predicted by the electroweak theory and of more general form, which might be induced by new physics. The parametrization used [10] only considers quartic terms in the Lagrangian which are not associated with TGCs, since the other terms are more strongly constrained by the measurements discussed in Section 2 and 3 Given the kinematic limit of LEP (maximum of two on-shell heavy bosons produced), the following vertices can be tested: $\mathrm{WW} \gamma \gamma$, parametrized by $a_{0}^{\mathrm{W}}$ and $a_{c}^{\mathrm{W}}$, WWZ $\gamma$, parametrized by $a_{n}$, and $\mathrm{ZZ} \gamma \gamma$ parametrized by $a_{0}^{\mathrm{Z}}$ and $a_{c}^{\mathrm{Z}}$. Only the first two vertices are present in the SM. Due to the dimension of Lagrangian operators containing four gauge bosons, quartic couplings have dimensions of the inverse of a squared energy and are there-

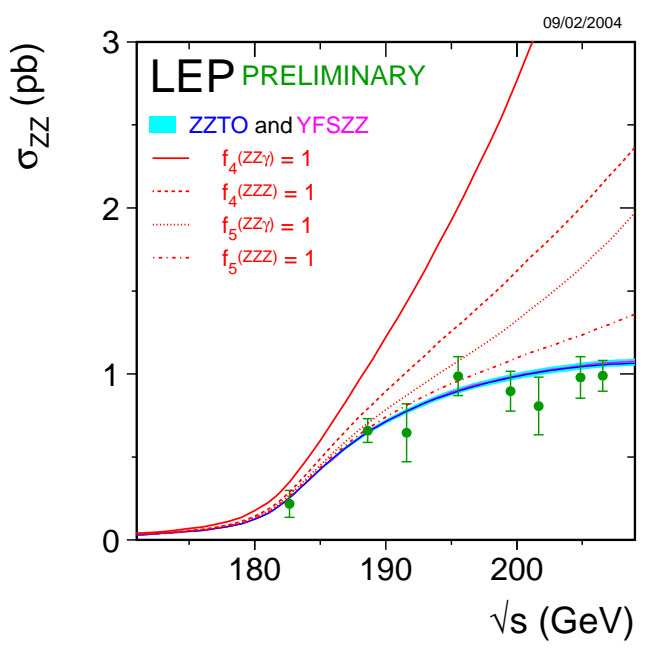

Figure 4. The LEP combined $\mathrm{e}^{+} \mathrm{e}^{-} \rightarrow \mathrm{ZZ}$ cross section. SM prediction and data points are shown together with curves corresponding to anomalous values of the $f_{4,5}^{\mathrm{V}}$ couplings.

fore normally written as proportional to $1 / \Lambda^{2}$, where $\Lambda$ is the typical energy scale of the new physics responsible for the QGCs.

At LEP, three topologies can give information about QGCs. The first one is $\mathrm{e}^{+} \mathrm{e}^{-} \rightarrow \mathrm{WW} \gamma$, which is sensitive to the couplings $a_{0}^{\mathrm{W}}, a_{c}^{\mathrm{W}}$ and $a_{n}$. Events with this topology are produced via radiative $\mathrm{W}$-pair production, where a photon is radiated from one of the initial or final state fermions or from one of the $\mathrm{W}$ bosons. Anomalous couplings would increase the cross section and modify the photon energy spectrum, as can be seen in Figure [5] where DELPHI data [1] is shown together with Monte Carlo expectations for SM and for some non-zero values of the coupling $a_{c}^{\mathrm{W}}$.

The second channel sensitive to QGCs is $\mathrm{e}^{+} \mathrm{e}^{-} \rightarrow \nu \bar{\nu} \gamma \gamma$. This final state can be produced both through $\mathrm{W}$ fusion into a $\mathrm{WW} \gamma \gamma$ vertex and via s-channel $\mathrm{Z}$ production, a $\mathrm{ZZ} \gamma \gamma$ vertex and subsequent decay of the $\mathrm{Z}$ into a neutrino pair. The same signature of two acoplanar photons is used in separate fits to determine the $\mathrm{W}$ and $\mathrm{Z}$ couplings, each time varying only one parame- 

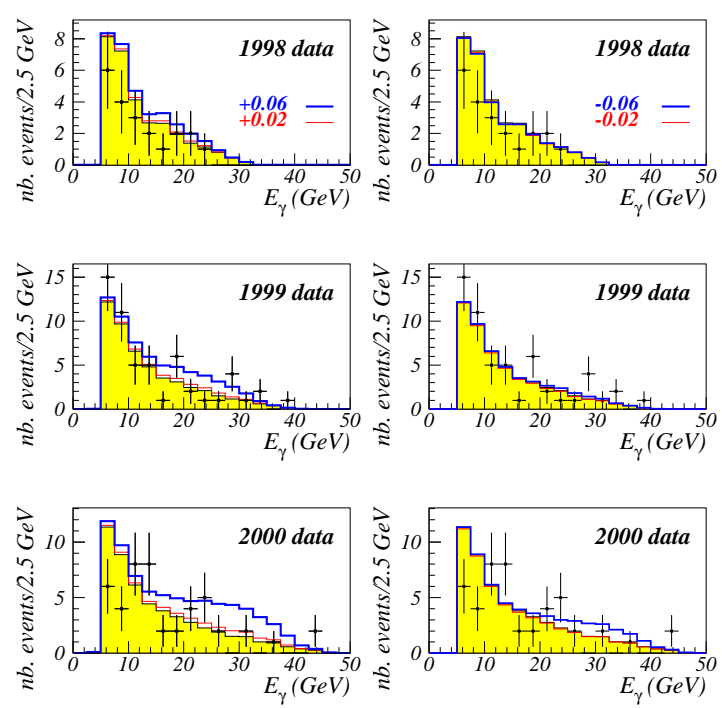

Figure 5. Photon energy spectrum in the $\mathrm{e}^{+} \mathrm{e}^{-} \rightarrow$ $\mathrm{WW} \gamma$ channel as measured by DELPHI. The three data sets correspond to $\sqrt{s}=189 \mathrm{GeV}$ (top), $\sqrt{s}=198 \mathrm{GeV}$ (middle) and $\sqrt{s}=206 \mathrm{GeV}$ (bottom). Data (dots) are compared to SM expectation (shaded histograms) and to the corresponding distributions for positive (left) and negative (right) values of $a_{c}^{\mathrm{W}}\left(\right.$ in $\left.\mathrm{GeV}^{-2}\right)$.

ter while setting all others to zero. Kinematic variables normally used in the fit are the recoil mass and the energy of the second highest energetic photon. In Figure 6 distributions of these variables as measured by OPAL 12 are plotted together with expectations for SM and for anomalous values of the parameters $a_{0}^{\mathrm{Z}}$ and $a_{0}^{\mathrm{W}}$. The ALEPH collaboration has also recently published [13] limits on QGCs extracted from this channel.

The $\mathrm{e}^{+} \mathrm{e}^{-} \rightarrow \mathrm{Z} \gamma \gamma \rightarrow \mathrm{q} \overline{\mathrm{q}} \gamma \gamma$ channel is also used at LEP to set limits on the $a_{0}^{Z}$ and $a_{c}^{Z}$ parameters. The corresponding SM background is the double radiative return to the $Z$. Quartic couplings of the $\mathrm{ZZ} \gamma \gamma$ type would enhance the total cross section at high $\sqrt{s}$ and modify the photons spectra. Figure 7 shows the L3 measurement of the $\mathrm{Z} \gamma \gamma$ cross
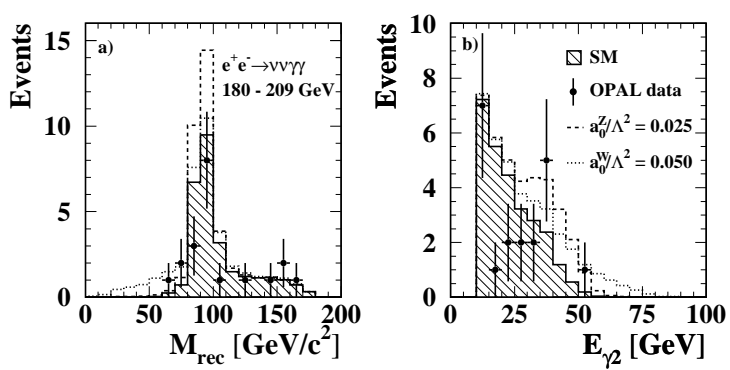

Figure 6. Recoil mass (left) and energy of the second highest energetic photon (right) for $\nu \bar{\nu} \gamma \gamma$ events collected by OPAL. Data and SM expectations are shown, together with predictions for non-zero values of $a_{0}^{\mathrm{Z}}$ and $a_{0}^{\mathrm{W}}$.

section as a function of centre-of-mass energy and the predicted behaviour in case of anomalous couplings.

The results of the combination of the ALEPH, L3 and OPAL measurements of the $\mathrm{ZZ} \gamma \gamma$ couplings are $-0.029 \mathrm{GeV}^{-2}<a_{c}^{\mathrm{Z}} / \Lambda^{2}<$ $+0.039 \mathrm{GeV}^{-2}$ and $-0.008 \mathrm{GeV}^{-2}<a_{0}^{Z} / \Lambda^{2}<$ $+0.021 \mathrm{GeV}^{-2}$ at $95 \%$ C.L., including statistical and systematic uncertainties. For the $\mathrm{WW} \gamma \gamma$ and $\mathrm{WWZ} \gamma$ couplings, no recent LEP combined results exist. The results of the single LEP experiments are reported in Table 3 .

\section{CONCLUSION}

Results of the measurements of triple and quartic gauge boson couplings by the four LEP experiments are reviewed in this paper, together with their combinations. Most results are either published or being finalized for publication. The measurement of charged TGCs, which are predicted as a fundamental consequence of the gauge structure of the electroweak theory, has reached at LEP the precision of about $2-4 \%$, with results in agreement with the SM. Searches for anomalous triple and quartic couplings have been performed by all LEP experiments in several different final states, and limits are set with no deviations observed from the SM predictions. 
Table 3

Measurements of $\mathrm{a}_{0}^{\mathrm{W}}, \mathrm{a}_{\mathrm{c}}^{\mathrm{W}}$ and $\mathrm{a}_{\mathrm{n}}$ by the LEP experiments. Limits are expressed in $\mathrm{GeV}^{-2}$ at $95 \%$ C.L.; statistical and systematic uncertainties are included.

\begin{tabular}{lcccc}
\hline parameter & ALEPH & DELPHI & L3 & OPAL \\
\hline $\mathrm{a}_{0}^{\mathrm{W}} / \Lambda^{2}$ & {$[-0.060,+0.055]$} & {$[-0.020,+0.020]$} & {$[-0.015,+0.015]$} & {$[-0.020,+0.020]$} \\
$\mathrm{a}_{\mathrm{c}}^{\mathrm{W}} / \Lambda^{2}$ & {$[-0.099,+0.093]$} & {$[-0.063,+0.032]$} & {$[-0.048,+0.026]$} & {$[-0.052,+0.037]$} \\
$\mathrm{a}_{\mathrm{n}} / \Lambda^{2}$ & - & {$[-0.18,+0.14]$} & {$[-0.14,+0.13]$} & {$[-0.16,+0.15]$} \\
\hline
\end{tabular}

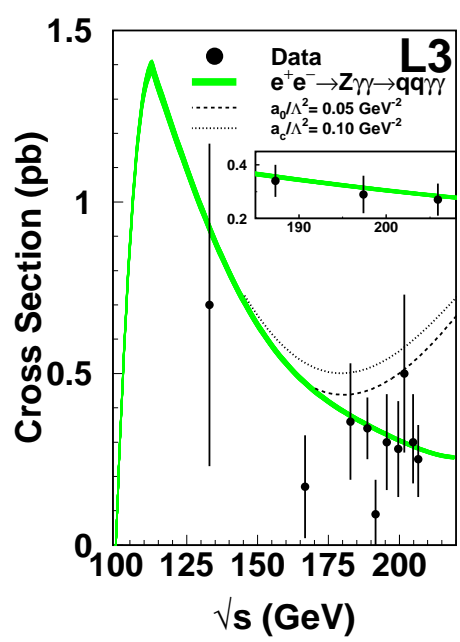

Figure 7. The L3 measurement of the cross section of the process $\mathrm{e}^{+} \mathrm{e}^{-} \rightarrow \mathrm{Z} \gamma \gamma \rightarrow \mathrm{q} \overline{\mathrm{q}} \gamma \gamma$ as a function of $\sqrt{s}$. Dashed and dotted lines represent anomalous QGC predictions. The inset presents the same results with the highest energy data combined in three samples.

\section{REFERENCES}

1. S.L. Glashow, Nucl. Phys. 22 (1961) 579; S. Weinberg, Phys. Rev. Lett. 19 (1967) 1264; A. Salam, in Elementary Particle Theory, ed. N. Svartholm, Stockholm, Almquist and Wiksell (1968), 367.

2. G.K. Fanourakis, D. Fassouliotis and S.E. Tzamarias, Nucl. Inst. Meth. A414 (1998), 399.

3. The LEP-TGC combination group, LEPEWWG/TGC/2003-01, (2004), avail- able at

http://www . cern. ch/LEPEWWG/lepww/tgc.

4. K. Hagiwara et al., Nucl. Phys. B282 (1987), 253.

5. K. Gaemers and G. Gounaris, Z. Phys. C1 (1979) 259; M. Bilenky et al., Nucl. Phys. B409 (1993) 22; Nucl. Phys. B419 (1994) 240; I. Kuss and D. Schildknecht, Phys. Lett. B383 (1996) 470.

6. G. Gounaris et al., in Physics at LEP 2, Report CERN 96-01 (1996), eds. G. Altarelli, T. Sjöstrand, F. Zwirner, Vol. 1, p. 525.

7. The L3 Collaboration, P. Achard et al., Phys. Lett. B586 (2004) 151.

8. G.J. Gounaris, J. Layssac and F.M. Renard, Phys. Rev. D62 (2000) 073013.

9. The L3 Collaboration, P. Achard et al., Phys. Lett. B597 (2004) 119.

10. G. Bélanger and F. Boudjema, Phys. Lett. B288 (1992) 201; J.W. Stirling and A. Werthenbach, Eur. Phys. J. C14 (2000) 103.

11. The DELPHI Collaboration, J. Abdallah et al., Eur. Phys. J. C31 (2003) 139.

12. The OPAL Collaboration, Constraints on Anomalous Quartic Gauge Boson Couplings from $\nu \bar{\nu} \gamma \gamma$ and $\mathrm{q} \overline{\mathrm{q}} \gamma \gamma$ Events at LEP2, CERNPH-EP-2004-003, submitted to Phys. Rev. D.

13. The ALEPH Collaboration, Constraints on anomalous $Q G C$ 's in $\mathrm{e}^{+} \mathrm{e}^{-}$interactions from 183 to $209 \mathrm{GeV}$, CERN-PH-EP-2004-001, submitted to Phys. Lett. B. 